\title{
Application of image processing and adaptive neuro-fuzzy system for estimation of the metallurgical parameters of a flotation process
}

\begin{abstract}
It is a well-known fact in the literature and practice that flotation froth features are closely related to process conditions and performance. The authors have already developed some reliable algorithms for measurement of the froth surface visual parameters such as bubble size distribution, froth color, velocity and stability. Furthermore, the metallurgical parameters of a laboratory flotation cell were successfully predicted from the extracted froth features. In this research study, the fuzzy c-mean clustering technique is utilized to classify the froth images (collected under different process conditions) based on the extracted visual characteristics. The classification of the images is actually necessary to determine the ideal froth structure and the target set-points for a machine vision control system. The results show that the captured froth images are well-classified into five categorizes on the basis of the extracted features. The correlation between the visual properties of froth (in different classes) and the metallurgical parameters is discussed and modeled by the adaptive neuro-fuzzy inference system (ANFIS). The promising results illustrate that the performance of the existing batch flotation system can be satisfactorily estimated from the measured froth characteristics. Therefore, the outputs from the current machine vision system can be inputted to a process control system.
\end{abstract}

Keyword: Froth flotation; Image classification; Image processing; Metallurgical parameters 